\title{
Prevalence and Predictors of Delayed Initiation of Breastfeeding Among Postnatal Women at a Tertiary Hospital in Eastern Uganda: a Cross- Sectional Study
}

\section{Loyce Kusasira}

busitema university

David Mukunya

Busitema University

Obakiro Samuel

Busitema University

Kiyimba Kenedy

Busitema University

Nekaka Rebecca

Busitema University

Lydia Ssenyonga

Busitema University

Mbwali Immaculate

Busitema University

Napyo Agnes ( $\square$ napyoagnes@gmail.com )

Busitema University https://orcid.org/0000-0003-4333-3588

\section{Research}

Keywords: delayed initiation of breastfeeding, early initiation of breastfeeding, breastfeeding, infants, lactating women

Posted Date: October 19th, 2021

DOl: https://doi.org/10.21203/rs.3.rs-968600/v1

License: (9) (1) This work is licensed under a Creative Commons Attribution 4.0 International License. Read Full License 


\section{Abstract}

\section{Background}

The rates for the delayed initiation of breastfeeding in Uganda remain unacceptably high and reasons for this are not well understood. We aimed to determine the prevalence and predictors for the delayed initiation of breastfeeding in Eastern Uganda.

\section{Methods}

This study employed a cross-sectional study design. A total of 404 mother-infant pairs were enrolled onto the study between July and November, 2020 at Mbale regional referral hospital (MRRH). They were interviewed on socio-demographic related, infant-related, labour and delivery characteristics using a structured questionnaire. We estimated adjusted odds ratios using multivariable logistic regression models.

\section{Results.}

The rate of delayed initiation of breastfeeding was $70 \%$ ( $n=283 / 404,95 \% \mathrm{Cl}: 65.3 \%-74.4 \%$ ). The factors that were associated with delayed initiation of breastfeeding were maternal charateristics including: being single (AOR $=0.37 ; 95 \% \mathrm{Cl}: 0.19-0.74)$, receiving antenatal care for less than 3 times while pregnant $(A O R=1.85,95 \% \mathrm{Cl}: 1.07-3.19)$ undergoing a caesarean section ( $\mathrm{AOR}=2.07 ; 95 \% \mathrm{Cl}: 1.3-3.19)$ and having a difficult labour (AOR=2.05; 95\% Cl: $1.25-3.35)$. Infant characteristics included: having a health issue at birth (AOR=9.8; 95\% Cl: $2.94-32.98)$.

\section{Conclusions:}

The proportion of infants that do not achieve early initiation of breastfeeding in this setting remains high. Women at high risk of delaying the initiation of breastfeeding include those who: deliver by caesarean section, do not receive antenatal care and have labour difficulties. Infants at risk of not achieving early initiation of breastfeeding include those that have a health issue at birth. We recommend increased support for women who undergo caesarean section in the early initiation of breastfeeding. Breastfeeding support can be initiated in the recovery room after caesarean delivery or in the operating theatre. The importance of antenatal care attendance should be emphasized during health education classes.

\section{Background}

The Uganda national policy guidelines on infant and young feeding recommend early initiation of breastfeeding (EIBF) within 1 hour of birth, exclusive breastfeeding for 6 months and there after continued breastfeeding for 2 years and beyond while introducing nutritionally adequate and age appropriate complementary foods [1]. Colostrum, the yellowish sticky breast milk produced at the end of pregnancy and first days after birth is the perfect food for the new born and this can be tapped through EIBF [2]. Colostrum is a rich source of nutrients, contains protective factors with anti-infective action, 
immunoglobulin, cytokines, complement-system components, leukocytes, oligosaccharides, nucleotides, lipids, and hormones that interact with each other and with the mucous membranes of the digestive and upper respiratory tracts of infants, providing passive immunity as well as stimulation for the development and maturation of the infant's immune system [3]. The antimicrobial factors present in colostrum and milk have some common characteristics, such as resistance to degradation by digestive enzymes, protection of the mucosal surfaces and elimination of bacteria without initiating inflammatory reactions [3]. Therefore infants who miss out on EIBF, will miss out on the colostrum and this puts them at risk of opportunistic infections like diarrhea as well as hospitalization $[4,5]$.

In addition, delayed initiation of breastfeeding has been associated with increased risk of neonatal death, caesarean section delivery, home delivery, maternal education, mother being overweight, antenatal care attendance, geographical location, HIV infection, skin-to-skin contact between mother and infant after birth [6-11]. Available, evidence has demonstrated that infants who start breastfeeding within 1 hour of birth will most likely exclusively breastfeed $[5,12]$.

The Ugandan government through the ministry of health has put up initiatives to promote ElBF like maternity-leave, provision of free antenatal care at public health facilities and health education on infant feeding during antenatal visits [1]. Despite these initiatives, the practice of EIBF in Uganda is divergent from the ideal practice that is recommended in the policy guidelines on infant and young child feeding $[1$, 12]. The rates of EIBF vary across and within countries $[7,8,12-14]$ and Uganda is no exception $[12,14-$ 16]. The reasons behind this discrepancy are not fully explored or understood and vary from context to context. There is also paucity of published evidence on this subject in Mbale city in Eastern Uganda. Given this background, it was vital for us to conduct this study to determine the prevalence and predictors for delayed initiation of breastfeeding in Eastern Uganda. The findings from this study will help in identifying groups of women and infants that are at risk of delaying the initiation to breastfeeding for their infants. These groups can then be a target for interventions of appropriate infant feeding counselling and support.

\section{Methods}

\section{Study design and setting}

This study employed a cross-sectional study design. Mother-infant pairs were enrolled onto the study between July and November, 2020 at Mbale regional referral hospital (MRRH), found in Mbale city, Eastern Uganda. MRRH is a general and teaching hospital for several medical and nursing institutions. It has a 400-bed capacity and serves over ten neighbouring districts in Mbale sub-region as a referral hospital. These include Kibuku, Kween, Bududa, Busia, Kapchorwa, Budaka, Butaleja, Pallisa, Manafwa, Namisindwa, Butebo, Sironko, Bukwo, Bulambuli and Tororo. However, the hospital also receives some patients from as far as Teso and Karamoja sub-regions. MRRH offers a wide range of health care services and these include: surgery, psychiatry, paediatric care, ear-nose and throat, outpatient, eye care, laboratory and dental care. This clinic also hosts special clinics such as gynaecology and obstetrics 
which include antenatal, maternity, postnatal and infant / young child care services. All these services are at no cost to the patients. In the Ugandan health care setting, pregnant women are free to seek antenatal care, labour and delivery services at any public health facility of their choice. However, during routine antenatal care classes at public health facilities, these women are advised to seek these services at health facilities that are nearest to where they reside. When the woman goes into labour, she reports to a public health facility and is admitted at the maternity ward. After delivery in the labour suite, women and their infants are then transferred to the postnatal ward where they both are monitored continuously for any complications that could arise. While admitted in the postnatal ward, women have to move to the young child clinic to take their infants for immunization. It is aslo possible that women-infant pairs registered and delivered from other health facilities other than MRRH are referred to the postnatal clinic for observation and to the young child clinic for immunization or child care. This study was conducted at the postnatal and young child clinics to obtain any information about the women and their infants.

\section{Participants and procedures}

We consecutively enrolled onto the study, mother-infant pairs who were receiving postnatal care and those attending the young child clinic at MRRH. We recruited women with infants who were aged $0-6$ days. We excluded women with infants aged 7 days or more for the purpose of mitigating recall bias. After consenting, women were interviewed on socio-demographic-related, infant-related characteristics as well as issues surrounding their labour and delivery. The information was collected using questionnaires through face-to-face interviews. The interviews were conducted in Luganda, Lumasaba (the languages predominantly spoken in the study setting) or English by trained research assistants. The questionnaires used during the interview process were administered by trained research assistants that were fluent in these local languages. The data collection tool was piloted to remove any irrelevant questions and avoid misinterpretation. The research assistants were qualified midwives who had experience in conducting research and providing postnatal care as well as immunization. While collecting data, research assistants had to implement COVID-19 preventive measures like use of face masks, wearing of plastic aprons, hand washing with soap and water. A total of 404 mother-infant pairs were included in the final analysis, Fig. 1.

\section{Study size}

The sample size used in this study was determined using the Cochran formular stated as $N=\left[\mathrm{Z}^{2} \mathrm{PQ}\right] / \mathrm{d}^{2}$ [17]. Where: $N$ is the sample size, the $Z$ - score is at a $95 \%$ confidence level is $1.96 . P$ is the prevalence of EIBF in Uganda at $65 \%$ [16]. Q is (1-p). $d$ is the acceptable margin of error which is $5 \%$ or 0.05 . We assumed a non-response rate of $10 \%$. The minimum sample size required for this study was 389 , we however included 404 postnatal women and their infants.

\section{Variables}

The outcome variable in our study was delayed initiation of breastfeeding defined as putting the infant on the breast to suckle beyond one hour after birth [1]. The exposures included in this study were maternal socio-demographic characteristics, labour- and delivery- related as well as infant-related characteristics. Socio-demographic characteristics included maternal age which was recorded as the 
completed number of years for each individual; marital status was categorised and labelled as "married" if the women reported to be cohabiting or married. If she was divorced, separated or living alone, she was categorised as "single". Religion was categorised as either "Christian" or "Moslem". All those women that were formally or self-employed were categorised and labelled as "employed" and the rest as "unemployed". Any residence that was estimated to be more than 10 kilometres from Mbale municipality was categorised and labelled as "rural" or else "urban". Women whose labour started between 06.00 hours to 18.59 hours were categorised as "day-time onset of labour" and else "night-time onset of labour". Women who delivered in any type of health care setting were all categorised as "hospital delivery" and otherwise as "non-hospital delivery". Women who had reported to have had a lot of bleeding after delivery, prolonged labour, an episiotomy, high blood pressure, retained placenta, perineal tears, cervix not opening, breech presentation and draining liquor were all categorised and labelled as "had labour difficulty" or else "none". Women who delivered their infants between 06.00 hours to 18.59 hours were categorised and labelled as having delivered in the "day-time" and else "night-time". An infant was categorised to have "had a health issue at birth" if the mother reported the infant to have weighed $>2.5$ $\mathrm{Kg}$, had difficulty in breathing, fever or diarrhoea. We, however, did not measure the body temperature of the infants due to the restrictive measures undertaken in MRRH for the prevention of SARS-CoV2. The age of the infants was recorded as completed number of days the infant had lived after birth.

\section{Data analysis and management}

Data were entered into excel software and exported for analysis into Stata version 14.0 (StataCorp, College Station, Texas, U.S.A.). Continuous data, if normally distributed, was summarised into means and standard deviations and if skewed, was summarised into medians with their corresponding interquartile ranges. Categorical variables were summarised into frequencies and percentages. The proportion of women who delayed to initiate breastfeeding was estimated and its confidence limits calculated using the exact method. We used multivariable generalized linear model regression analysis with a logit link to estimate the adjusted odds ratios of the independent variables on delayed initiation of breastfeeding while controlling for confounding. All variables with $p<0.25$ at the bivariate level were included in the initial model at the multivariate analysis. All variables with $p<0.1$ and those of biological or epidemiologic plausibility (from previous studies) were included in the second model. We relied on the likelihood-ratio test to check the significant difference between the initial and the second model. There was no difference between the two models so then we adapted the second model.

\section{Results}

\section{Prevalence of early inintiation of breastfeeding}

In our study, $70 \%(\mathrm{n}=283 / 404,95 \% \mathrm{Cl}: 65.3 \%-74.4 \%)$ of the postnatal women did not breastfeed their infants within one hour after birth.

\section{Sociodemographic-related characteristics}


The median age of women attending the postnatal clinic at MRRH was 24 years (IQR 20,30). The majority of these women were married $(87.1 \%, \mathrm{n}=221 / 404)$, Christian $(66.8 \%)$, unemployed $(75.5 \%)$ and lived in a rural residence $(71.5 \%)$. Only $10 \%$ of the women had received a tertiary education. Most of the women $(70 \%)$ had 3 or more antenatal visits while they were pregnant. About fourty percent $(n=156 / 404)$ had a difficult labour. More than half of the women delivered by cesarean section (57.2\%) and in a hospital setting (98.3\%). Many of the infants born to these women had no health issue at birth (84.9\%).

Approximately fifty percent of the infants born to these women were male $(n=206 / 404)$ and their mean weight was $3.21 \mathrm{Kg}$ (SD 0.62). The median age of these infants was 1 day (IQR 1,2). (Table 1). 
Table 1

Characteristics of mothers and their infants

\begin{tabular}{|c|c|c|c|c|}
\hline \multirow[b]{2}{*}{ Variable } & \multicolumn{4}{|c|}{ Early initiation of breastfeeding } \\
\hline & No & Yes & Total (N) & P-value \\
\hline & $\mathrm{N}=283(70)$ & $\mathrm{N}=121(30)$ & $\mathrm{N}=404$ & \\
\hline & $N(\%)$ & $N(\%)$ & & \\
\hline \multicolumn{5}{|c|}{ Mothers' individual characteristics } \\
\hline \multicolumn{5}{|l|}{ Age (years) } \\
\hline$<20$ & $55(19.4)$ & $26(21.5)$ & $81(20.0)$ & 0.766 \\
\hline $20-29$ & $154(54.4)$ & $67(54.4)$ & $221(54.7)$ & \\
\hline$\geq 30$ & $74(26.2)$ & $28(23.1)$ & $102(25.3)$ & 0.599 \\
\hline \multicolumn{5}{|l|}{ Marital status } \\
\hline Married & $253(89.4)$ & $99(81.8)$ & $352(87.1)$ & \\
\hline Single & $30(10.6)$ & $22(18.2)$ & $52(12.9)$ & 0.039 \\
\hline \multicolumn{5}{|l|}{ Religion } \\
\hline Christian & 195(68.9) & $75(62.0)$ & $270(66.8)$ & \\
\hline Moslem & $88(31.1)$ & $46(38.0)$ & $134(33.2)$ & 0.177 \\
\hline \multicolumn{5}{|l|}{ Education level } \\
\hline Primary & $134(47.3)$ & $48(39.7)$ & $182(45.0)$ & \\
\hline Secondary & $121(42.8)$ & $61(50.4)$ & $182(45.0)$ & 0.138 \\
\hline Tertiary & 28(9.9) & $12(9.9)$ & $40(10.0)$ & 0.64 \\
\hline \multicolumn{5}{|c|}{ Employment status } \\
\hline Not employed & $219(77.4)$ & $86(71.1)$ & $305(75.5)$ & \\
\hline Employed & $64(22.6)$ & $35(28.9)$ & $99(24.5)$ & 0.178 \\
\hline \multicolumn{5}{|l|}{ Residence } \\
\hline Urban & $72(25.4)$ & $43(35.5)$ & $115(28.5)$ & \\
\hline Rural & $211(74.6)$ & $78(64.5)$ & $289(71.5)$ & 0.04 \\
\hline \multicolumn{5}{|c|}{ Characteristics of labour and delivery } \\
\hline Number of AN & & & & \\
\hline
\end{tabular}




\begin{tabular}{|c|c|c|c|c|}
\hline \multirow[b]{2}{*}{$\geq 3$} & \multicolumn{4}{|c|}{ Early initiation of breastfeeding } \\
\hline & $191(67.5)$ & $94(77.7)$ & $285(70.5)$ & \\
\hline$<3$ & $92(32.5)$ & $27(22.3)$ & $119(29.5)$ & 0.041 \\
\hline \multicolumn{5}{|l|}{ Labour onset } \\
\hline Day-time & $126(44.5)$ & $59(48.8)$ & $185(45.8)$ & \\
\hline Night-time & $130(45.9)$ & $51(42.2)$ & $181(44.8)$ & 0.439 \\
\hline No pains & $27(9.6)$ & 11(9.0) & $38(9.4)$ & 0.722 \\
\hline \multicolumn{5}{|l|}{ Attendant in Labour } \\
\hline Husband & $58(20.5)$ & 23(19.0) & $81(20.1)$ & \\
\hline Other & $225(79.5)$ & $98(81.0)$ & $323(79.9)$ & 0.733 \\
\hline \multicolumn{5}{|l|}{ Labour difficulties } \\
\hline None & $161(56.9)$ & $87(71.9)$ & $248(61.4)$ & \\
\hline Had labour difficulty & $122(43.1)$ & $34(28.1)$ & 156(38.6) & 0.005 \\
\hline \multicolumn{5}{|l|}{ Mode of delivery } \\
\hline Normal delivery & 105(37.1) & $68(56.2)$ & $173(42.8)$ & \\
\hline Caesarean section & $178(62.9)$ & $53(43.8)$ & $231(57.2)$ & 0.000 \\
\hline \multicolumn{5}{|l|}{ Time of delivery } \\
\hline Day time & $162(57.2)$ & $62(51.2)$ & $224(55.5)$ & \\
\hline Night time & $121(42.8)$ & $59(48.8)$ & $180(44.5)$ & 0.267 \\
\hline \multicolumn{5}{|l|}{ Place of delivery } \\
\hline Hospital & 277(97.9) & 120(99.2) & $397(98.3)$ & \\
\hline Non-hospital & $6(2.1)$ & $1(0.8)$ & $7(1.7)$ & 0.379 \\
\hline \multicolumn{5}{|c|}{ Characteristics of the infants } \\
\hline \multicolumn{5}{|l|}{ Infant's gender } \\
\hline Male & $146(51.6)$ & $60(49.6)$ & $206(51.0)$ & \\
\hline Female & $137(48.4)$ & $61(50.4)$ & 198(49.0) & 0.712 \\
\hline \multicolumn{5}{|l|}{ Infant's weight } \\
\hline Less than $2.5 \mathrm{~kg}$ & $29(10.3)$ & $5(4.1)$ & $34(8.4)$ & 0.049 \\
\hline $2.5 \mathrm{~kg}$ to $4.5 \mathrm{~kg}$ & $248(87.6)$ & 114(94.2) & $362(89.6)$ & \\
\hline
\end{tabular}




\begin{tabular}{|c|c|c|c|c|}
\hline \multirow[b]{2}{*}{ Greater than $4.5 \mathrm{~kg}$} & \multicolumn{4}{|c|}{ Early initiation of breastfeeding } \\
\hline & $6(2.1)$ & $2(1.7)$ & $8(2.0)$ & 0.697 \\
\hline \multicolumn{5}{|l|}{ Infant's age } \\
\hline Less than 3 days & $205(72.4)$ & 104(85.9) & $309(76.5)$ & \\
\hline Greater or equal to three days & $78(27.6)$ & $17(14.1)$ & $95(23.5)$ & 0.04 \\
\hline \multicolumn{5}{|l|}{ Birth order } \\
\hline 1 & $102(36.0)$ & $46(36.0)$ & 148(36.6) & \\
\hline 2 & $59(20.9)$ & 24(19.8) & $83(20.5)$ & 0.731 \\
\hline 3 & $122(43.1)$ & $51(42.2)$ & $173(42.9)$ & 0.755 \\
\hline \multicolumn{5}{|l|}{ Infant had a health issue at birth } \\
\hline No & $225(79.5)$ & 118(97.5) & $343(84.9)$ & \\
\hline Yes & $58(20.5)$ & $3(2.5)$ & $61(15.1)$ & 0.000 \\
\hline
\end{tabular}

Women who were single were more likely to initiate breastfeeding within 1 hour of birth (AOR=0.37; $95 \% \mathrm{Cl}: 0.19-0.74)$ compared to those who are married. Women who had received antenatal care less than 3 times when pregnant were more likely to delay the initiation of breastfeeding $(A O R=1.85,95 \% \mathrm{Cl}$ : $1.07-3.19)$ compared to those that had received antenatal care 3 or more times. Women who underwent a caesarean delivery were less likely to initiate breastfeeding early (AOR=2.07; 95\% Cl: $1.3-3.19$ ) compared to women who had a spontaneous vaginal delivery. Women who had a difficult labour were more likely to delay the initiation of breastfeeding (AOR=2.05; $95 \% \mathrm{Cl}: 1.25-3.35)$ compared to those that did not have any difficulty during labour. Infants that had a health issue at birth were less likely to start breastfeeding within 1 hour after birth (AOR=9.8; 95\% Cl: $2.94-32.98)$ when compared with those that did not have a health problem (Table 2). 
Table 2

Factors influencing early initiation of breastfeeding

\begin{tabular}{|llc|}
\hline Variable & Unadjusted OR & Adjusted OR \\
\hline Age (years) & & \\
\hline$<20$ & $0.92(0.53-1.59)$ & $0.84(0.45-1.57)$ \\
\hline $20-29$ & 1 & 1 \\
\hline$\geq 30$ & $1.15(0.68-1.94)$ & $1.07(0.61-1.87)$ \\
\hline Marital status & & \\
\hline Married & 1 & 1 \\
\hline Single & $0.53(0.29-0.97)$ & $\mathbf{0 . 3 7}(\mathbf{0 . 1 9 - 0 . 7 4 )}$ \\
\hline Baby had a health issue at birth & \\
\hline No & 1 & 1 \\
\hline Yes & $10.14(3.11-33.05)$ & $9.8(2.94-32.98)$ \\
\hline Number of ANC visits & & \\
\hline$\geq 3$ & 1 & 1 \\
\hline$<3$ & $1.68(1.02-2.75)$ & $1.85(1.07-3.19)$ \\
\hline Mode of delivery & & 1 \\
\hline Normal delivery & 1 & $2.05(1.25-3.35)$ \\
\hline Caesarean section & $2.18(1.41-3.35)$ & $2.07(1.3-3.29)$ \\
\hline Labour difficulties & & \\
\hline None & 1 & \\
\hline Had labour difficulty & $1.94(1.22-3.07)$ & \\
\hline
\end{tabular}

\section{Discussion}

In this study we found that an unacceptably high proportion of women delayed the initiation of breastfeeding after birth. The rates of ElBF vary between and within countries ranging from $30-83 \%[7,8$, $20,9,10,12-15,18,19]$. Reasons for these findings also vary across contexts. More than half of the women in this cohort underwent caesarean section delivery. Several studies explain that this mode of delivery is associated with delayed initiation of breastfeeding as explained in the following paragraph. This could possibly explain the high rate of delayed initiation of breastfeeding among women in our study cohort. A considerable number of women in this study also had some form of labour difficulty. A number of infants in this study also had a health issue after birth. Health complications that arise during 
delivery among women and their infants also affect early initiation of breastfeeding and could have contributed to the high rate of non-EIBF in this study.

Consistent with studies done elsewhere $[7,8,22,23,9,10,13,14,18-21]$, our study found that delivery by caesarean section increased the odds of delayed initiation of breastfeeding. Along with caesarean delivery, comes exhaustion arising from the procedure itself and the effects of anaesthesia which may impede the early initiation of breastfeeding. A caesarean section birth takes a lot of time involving the repair of surgical incisions and recovery which may contribute to late breastfeeding initiation. Also many of the infants in this study that had a health issue at birth were actually delivered by caesarean delivery. These infants are likely to have difficulty in sucking. The combination of a caesarean delivery and the baby having a health issue at birth may further contribute to the risk of late breastfeeding initiation. Mothers who had undergone caesarean section may be less likely to introduce their new born infants (who may also have difficulty sucking) to breastfeeding within the recommended one hour after birth.

We found that infants that had a health issue at birth were more likely to delay to start breastfeeding after birth. This finding is consistent with evidence found in other studies [24,25]. These health issues included difficulty in breathing, fever, diarrhoea and the infant being too weak. Infants with health issues at birth may cause the infant to have difficulty in suckling due to weak breastfeeding reflexes, poor coordination and lack of ability to swallow. This may contribute to the delayed initiation of breastfeeding. In addition, the majority of the infants that had a health issue at birth had actually been born by caesarean delivery. This mode of delivery could have contributed to the delay in the initiation of breastfeeding as explained earlier.

Women who had received antenatal care less than 3 times while they were pregnant were less likely to initiate breastfeeding within 1 hour after birth. This association has been demonstrated in other studies $[8,12,22,24,26]$. During antenatal care, the benefits of EIBF are always emphasized in health education talks in MRRH. The more the antenatal care visits the women have, the more the interface they make with these health education talks. In this way, these women become more conversant with these counselling messages and are therefore more likely to support their infants in initiating breastfeeding within 1 hour after birth.

Women who had a difficult labour were more likely to delay the initiation of breastfeeding. This finding is consistent with evidence found elsewhere [8, 23, 27]. Difficult labour in our study included prolonged labour, body weakness, experiencing a lot of pain, prolonged bleeding and having received an episiotomy. Most of the women that had a difficult labour actually ended up giving birth by caesarean section. This mode of delivery could have contributed to the delay in EIBF as explained in earlier paragraphs. In addition, maternal and foetal indications for caesarean delivery and postoperative care disrupt bonding and mother-infant interaction and delay initiation of breastfeeding.

It is surprising that in our study, women who were single were more likely to practice EIBF. Other studies $[13,24]$ have found contrary evidence to ours. We recommend that a qualitative study can be conducted on this subject matter to best understand the occurrence of this association in our context. 


\section{Strengths And Limitations}

This study was done in a regional referral hospital. Women who deliver at this hospital are probably referred from other lower cadre health facilities due to complications in pregnancy. Our study findings may only be generalizable to this nature of population or those similar to it. We never asked any questions on cultural practices that may influence EIBF or why the infants were initiated late. This study had some strength, too. We conducted this study among infants that did not exceed 6 days of age. This recall period could have helped to counteract the possibility of recall bias. This study is the first to be published concerning EIBF in Mbale city and is of importance to healthcare service providers in this setting because it will help them to refocus interventions to the most affected groups of women and infants

\section{Conclusions and recommendations}

The proportion of infants that do not achieve EIBF in this setting remains unacceptably high at $70 \%$. Women at high risk of delaying the initiation of breastfeeding include those who: deliver by caesarean section, do not receive antenatal care and have labour difficulties. Infants at risk of not achieving EIBF include those that have a health issue at birth. We recommend increased support in the early initiation of breastfeeding for women who undergo caesarean section. Breastfeeding support can be initiated in the recovery room after caesarean delivery or in the operating theatre. The importance of antenatal care attendance should be emphasized during health education classes. We also recommend a qualitative investigation into the reasons as to why women in this setting delay to initiate breastfeeding for their newly born infants.

\section{Abbreviations}

AOR: Adjusted odds ratio

Cl: Confidence interval

COVID-19: Coronavirus disease of 2019.

EIBF: Early initiation of breastfeeding

MRRH : Mbale regional referral hospital

SARS-CoV2: Severe acute respiratory syndrome coronavirus 2

\section{Declarations}

\section{Ethical considerations}


Approval to conduct this study was obtained from the MRRH - research and ethics committee (reference number: MRRH-REC OUT 016/2020). Informed consent was obtained prior to data collection. The mother was not penalised or denied maternity or infant care if she declined participation. Identification numbers were used to ensure anonymity and no linkage to the clinic data of the participants. The data collected was kept confidential and only accessed by persons directly involved in the study.

\section{Consent for publication}

Not applicable

\section{Availability of data and materials}

The datasets used and/or analysed during the current study are available from the corresponding author on reasonable request.

\section{Competing interests}

The authors declare that they have no competing interests

\section{Funding}

Research reported in this publication was supported by the Fogarty International Centre of the National Institutes of Health, U.S. Department of State's Office of the U.S. Global AIDS Coordinator and Health Diplomacy (S/GAC), and President's Emergency Plan for AIDS Relief (PEPFAR) under award number 1R25TW011213. The content is solely the responsibility of the authors and does not necessarily represent the official views of the National Institutes of Health. The funders had no role in study design, data collection and analysis, decision to publish or preparation of the manuscript.

\section{Authors' contribution}

Conceptualization by LK, AN; Data curation by LK, AN; Formal analysis by LK, AN; Funding acquisition by NR; Methodology by LK, AN; Project administration by NR, LS; Resources by NR, LS; Supervision by AN; Writing of original draft by LK, AN, DM, OS, KK, Ml; Review and editing by LK, AN, DM, OS, KK, MI

All authors read and approved the final manuscript

\section{Acknowledgements}


We are grateful to Mbale Regional Referral Hospital, the study participants and the research assistants for their contribution to this survey.

\section{References}

1. Uganda M of H. Policy guidelines on infant and young child feeding. 2009.

2. World Health Organization (WHO). Infant and young child feeding: a tool for assessing national practices, policies and programmes. 2003.

3. Palmeira P, Carneiro-Sampaio M. Immunology of breast milk. Rev Assoc Med Bras. 2016;62:584-93. https://doi.org/10.1590/1806-9282.62.06.584.

4. Edmond KM, Zandoh C, Quigley MA, Amenga-Etego S, Owusu-Agyei S, Kirkwood BR. Delayed breastfeeding initiation increases risk of neonatal mortality. Pediatrics. 2006;117:e380-6. https://doi.org/10.1542/peds.2005-1496.

5. Paramashanti BA, Hadi H, Alit Gunawan IM. Timely initiation of breastfeeding is associated with the practice of exclusive breastfeeding in Indonesia. Asia Pac J Clin Nutr. 2016;25:52-6. https://doi.org/10.6133/apjcn.122016.s11.

6. Abdullah A, Hort K, Butu Y, Simpson L. Risk factors associated with neonatal deaths: A matched case-control study in Indonesia. Glob Health Action. 2016;9:30445. https://doi.org/10.3402/gha.v9.30445.

7. Adewuyi EO, Zhao Y, Khanal V, Auta A, Bulndi LB. Rural-urban differences on the rates and factors associated with early initiation of breastfeeding in Nigeria: further analysis of the Nigeria demographic and health survey, 2013. Int Breastfeed J. 2017;12:51-61. https://doi.org/10.1186/s13006-017-0141-x.

8. Ariful Islam M, Mamun A, Murad Hossain M, Bharati P, Saw A, Lestrel PE, et al. Prevalence and factors associated with early initiation of breastfeeding among Bangladeshi mothers: a nationwide cross-sectional study. PLoS One. 2019;14:e0215733. https://doi.org/10.1371/journal.pone.0215733.

9. Duodu PA, Duah HO, Dzomeku VM, Boamah Mensah AB, Aboagye Mensah J, Darkwah E, et al. Consistency of the determinants of early initiation of breastfeeding in Ghana: insights from four demographic and health survey datasets. Int Health. 2021;13:39-48. https://doi.org/10.1093/inthealth/ihaa017.

10. Nkoka O, Ntenda PAM, Kanje V, Milanzi EB, Arora A. Determinants of timely initiation of breast milk and exclusive breastfeeding in Malawi: A population-based cross-sectional study. Int Breastfeed J. 2019;14:37-45. https://doi.org/10.1186/s13006-019-0232-y.

11. Singh K, Khan SM, Carvajal-Aguirre L, Brodish P, Amouzou A, Moran A. The importance of skin-to-skin contact for early initiation of breastfeeding in Nigeria and Bangladesh. J Glob Health. 2017;7:020505. https://doi.org/10.7189/jogh.07.020505.

12. Bbaale E. Determinants of early initiation, exclusiveness, and duration of breastfeeding in Uganda. J Heal Popul Nutr. 2014;32:249-60. https://doi.org/10.3329/jhpn.v32i2.2619. 
13. Bruno Tongun J, Sebit MB, Mukunya D, Ndeezi G, Nankabirwa V, Tylleskar T, et al. Factors associated with delayed initiation of breastfeeding: a cross-sectional study in South Sudan. Int Breastfeed J. 2018;13:28-34. https://doi.org/10.1186/s13006-018-0170-0.

14. Mukunya D, Tumwine JK, Nankabirwa V, Ndeezi G, Odongo I, Tumuhamye J, et al. Factors associated with delayed initiation of breastfeeding: a survey in Northern Uganda. Glob Health Action. 2017;10:1410975. https://doi.org/10.1080/16549716.2017.1410975.

15. Kalisa R, Malande O, Nankunda J, Tumwine JK. Magnitude and factors associated with delayed initiation of breastfeeding among mothers who deliver in Mulago hospital. Uganda Afr Health Sci. 2015;15:1130-5. https://doi.org/10.4314/ahs.v15i4.11.

16. Uganda Bureau of Statistics. Uganda Demographic and Health Survey 2016. 2016.

17. Bartlett IIJE, Kotrlik JW, Higgins CC. Determing appropriate sample size in survey research. Inf Technol Learn Perform J. 2001;19:43-50.

18. Liben ML, Yesuf EM. Determinants of early initiation of breastfeeding in Amibara district, Northeastern Ethiopia: a community based cross-sectional study. Int Breastfeed J. 2016;11:7-13. https://doi.org/10.1186/s13006-016-0067-8.

19. Lyellu HY, Hussein TH, Wandel M, Stray-Pedersen B, Mgongo M, Msuya SE. Prevalence and factors associated with early initiation of breastfeeding among women in Moshi municipal, Northern Tanzania. BMC Pregnancy Childbirth. 2020;20:285-94. https://doi.org/10.1186/s12884-020-029660 .

20. Mukora-Mutseyekwa F, Gunguwo H, Mandigo RG, Mundagowa P. Predictors of early initiation of breastfeeding among Zimbabwean women: secondary analysis of ZDHS 2015. Matern Heal Neonatol Perinatol. 2019;5:2-8. https://doi.org/10.1186/s40748-018-0097-x.

21. Seidu AA, Ahinkorah BO, Agbaglo E, Dadzie LK, Tetteh JK, Ameyaw EK, et al. Determinants of early initiation of breastfeeding in Papua New Guinea: a population-based study using the 2016-2018 demographic and health survey data. Arch Public Heal. 2020;78:124-34. https://doi.org/10.1186/s13690-020-00506-y.

22. Shiferaw BZ. Factors associated with early initiation and exclusive breastfeeding practices among mothers of infant's age less than 6 months. J Pediatr Neonatal Care. 2017;7:00292. https://doi.org/10.15406/jpnc.2017.07.00292.

23. Takahashi K, Ganchimeg T, Ota E, Vogel JP, Souza JP, Laopaiboon M, et al. Prevalence of early initiation of breastfeeding and determinants of delayed initiation of breastfeeding: Secondary analysis of the WHO Global Survey. Sci Rep. 2017;7:44868. https://doi.org/10.1038/srep44868.

24. Ndirangu MN, Gatimu SM, Mwinyi HM, Kibiwott DC. Trends and factors associated with early initiation of breastfeeding in Namibia: analysis of the demographic and health surveys 2000-2013. BMC Pregnancy Childbirth. 2018;18:171-80. https://doi.org/10.1186/s12884-018-1811-4.

25. Sharma A, Thakur P, Tiwari R, Kasar P, Sharma R, Kabirpanthi V. Factors associated with early initiation of breastfeeding among mothers of tribal area of Madhya Pradesh, India: a community 
based cross sectional study. Int J Community Med Public Heal. 2016;3:194-9.

https://doi.org/10.18203/2394-6040.ijcmph20151561.

26. Woldeamanuel BT. Trends and factors associated to early initiation of breastfeeding, exclusive breastfeeding and duration of breastfeeding in Ethiopia: evidence from the Ethiopia demographic and health urvey 2016. Int Breastfeed J. 2020;15:3-15. https://doi.org/10.1186/s13006-019-0248-3.

27. Sharma IK, Byrne A. Early initiation of breastfeeding: a systematic literature review of factors and barriers in South Asia. Int Breastfeed J. 2016;11:17-28. https://doi.org/10.1186/s13006-016-0076-7.

Figures

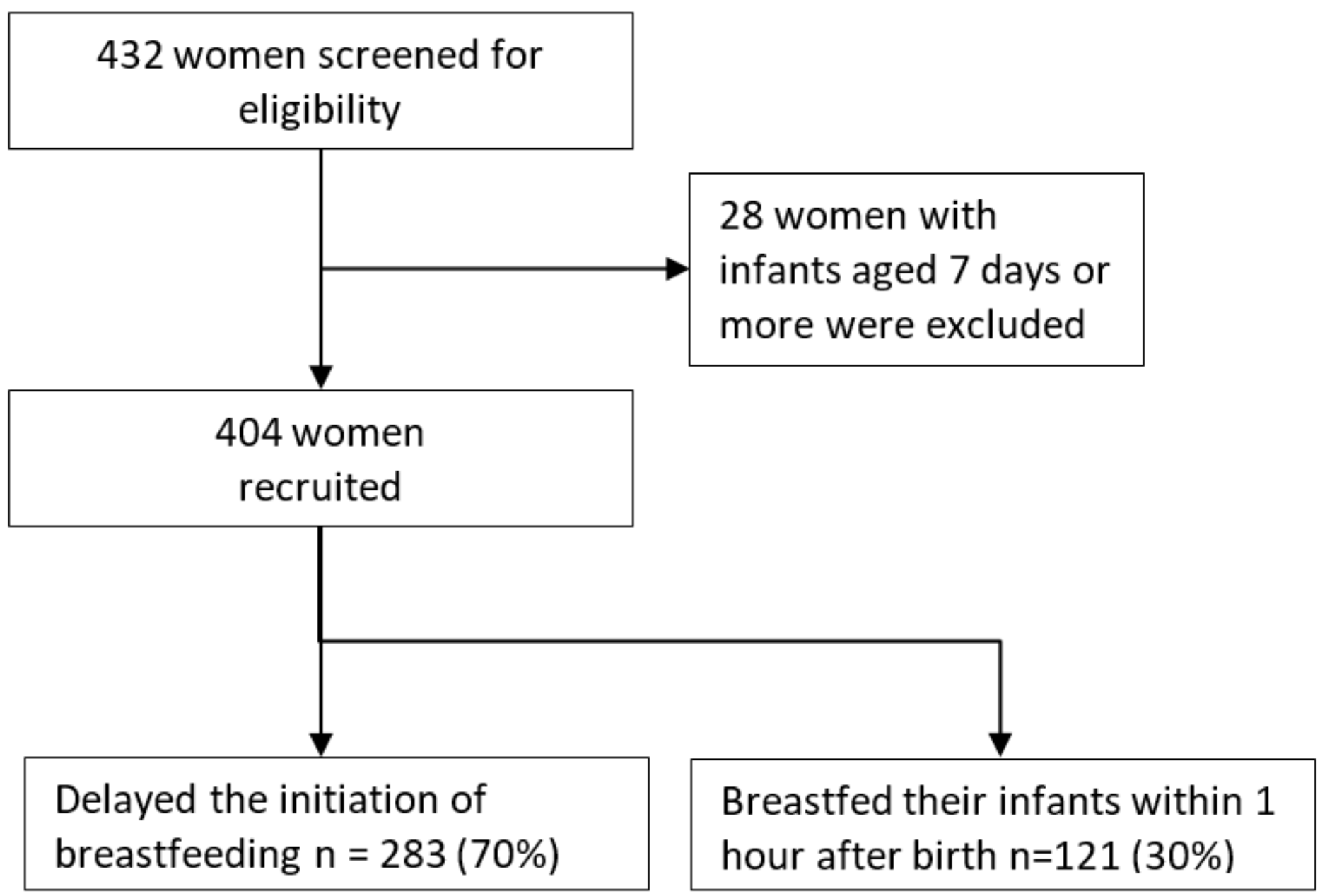

Figure 1

Study flow chart 